\title{
A Non-linear Feedback Neural Network for Graph Coloring
}

\author{
Mohd. Samar Ansari \\ Department of Electronics \\ Engineering \\ Aligarh Muslim University \\ Aligarh, India
}

\author{
Syed Atiqur Rahman \\ Department of Electronics \\ Engineering \\ Aligarh Muslim University \\ Aligarh, India
}

\author{
Syed Javed Arif \\ Department of Electronics \\ Engineering \\ Aligarh Muslim University \\ Aligarh, India
}

\begin{abstract}
A feedback neural network for solving graph coloring problem is presented. The circuit has an associated transcendental energy function that ensures fast convergence to the exact solution. Hardware and PSPICE simulation results on random and benchmark problems have been presented. Test results are compared with existing techniques for graph coloring to show that the proposed neural network model provides a significant reduction in the number of colors while enjoying a simple and efficient circuit implementation.
\end{abstract}

\section{General Terms}

Neural Networks, Graph Coloring.

\section{Keywords}

Neural network applications, Neural network hardware, Nonlinear circuits, Graph theory,Dynamical Systems, Non-Linear Synapse,Feedback Networks.

\section{INTRODUCTION}

The graph coloring problem (GCP) has a large number of applications such as register allocation in digital computers, frequency channel assignment in mobile communication, and layer assignment in VLSI design [1-3]. In GCP, values or colors are assigned to different nodes of a graph such that no two adjacent nodes get the same color, and the number of colors are also minimized. The Hopfield Neural Network (HNN) and its modifications have been applied to solve GCP, but the implementation requires $\mathrm{N}^{2}$ neurons and at most $\mathrm{N}^{4}$ weights for coloring a graph of $\mathrm{N}$ nodes [4]. This puts severe constraints on the feasibility of HNN realization in hardware. Another feedback neural network architecture which uses non-linear synapses, and has a transcendental Energy function, requires $\mathrm{N}$ neurons and at most $\mathrm{N}^{2}$ interconnections for solving a problem with $\mathrm{N}$ nodes [4]. In this paper, a neural circuit is presented which modifies the Energy function of [4] to eliminate those local minima that correspond to solutions with large number of colors. It is seen that the number of colors are reduced thereby providing a significant improvement in solution quality. Moreover, the circuit is suitable for VLSI implementation as compared to an existing scheme which employs one coupled relaxation oscillator for each node in the graph to be colored [5].

\section{PROPOSED CIRCUIT FOR GRAPH COLORING}

Fig. 1 shows thei-th neuron of proposed network. In the proposed circuit, output voltages of different neurons represent the colors of different nodes. $\mathrm{C}_{\mathrm{i}}$ and $\mathrm{r}_{\mathrm{i}}$ denote the internal capacitance and resistance of the $i$-th neuron respectively, $u_{i}$ is the internal state and $R_{\mathrm{ii}}$ is the self-feedback resistance of $i$-th neuron. The output of other neurons $\mathrm{V}_{\mathrm{j}}(\mathrm{j}=$ $1,2 \ldots \mathrm{N})$ are connected to the input of $i$-th neuron through unipolar comparators.

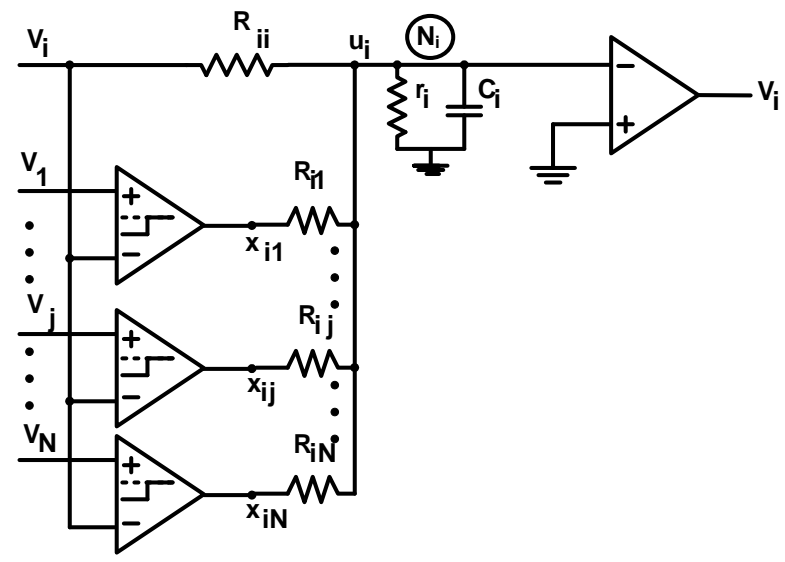

Fig 1: $i$-th neuron of the proposed neural network for graph coloring

The self - feedback resistance $\left(R_{i i}\right)$ is given by

$$
R_{i i}=\frac{R_{c}}{\Delta}
$$

where $R_{c}$ is a constant resistance and $\Delta$ is the maximum degree of the graph. Next, we define a constant $a_{i j}$ such that

$$
a_{i j}=\left\{\begin{array}{cc}
1 ; & i-\text { th node connected to } j-\text { th node } \\
0 ; & \text { otherwise }
\end{array}\right\}
$$

Using (2), the feedback resistance of the $i$-th neuron is given by

$$
R_{i j}=\frac{R_{c}}{a_{i j}}
$$

From fig. $1, \mathrm{x}_{\mathrm{ij}}$ can be written as

$$
x_{i j}=\frac{V_{m}}{2}\left[\tanh \beta\left(V_{j}-V_{i}\right)-1\right]
$$

where $\beta$ is the open-loop gain of the comparator (practically very high) and $\pm \mathrm{V}_{\mathrm{m}}$ are the voltage levels of the comparator output.

Node equation for node ' $\mathrm{N}_{\mathrm{i}}$ ' gives the equation of motion of the $i$-th neuron in the state space as

$$
C_{i} \frac{d u_{i}}{d t}=\sum_{\substack{j=1 \\ j \neq i}}^{N} \frac{x_{i j}}{R_{i j}}+\frac{V_{i}}{R_{i i}}-\frac{u_{i}}{R_{i}}(5)
$$


where

$$
\frac{1}{R_{i}}=\sum_{\substack{j=1 \\ j \neq i}}^{N} \frac{1}{R_{i j}}+\frac{1}{R_{i i}}+\frac{1}{r_{i}}(6)
$$

Moreover, it can be shown that the Energy Function ' $E$ ' of the network in Fig. 1 is

$E=\frac{1}{2} \sum_{i=1}^{N} \frac{V_{i}{ }^{2}}{2 R_{i i}}-\frac{V_{m}}{4 \beta R_{c}} \sum_{i=1}^{N} \sum_{j=1}^{N} a_{i j} \ln \cosh \left(\beta\left(V_{j}-V_{i}\right)\right)-$ $\frac{V_{m}}{2 R_{c}} \sum_{i=1}^{N} \sum_{\substack{j=1 \\ j \neq i}}^{N} a_{i j} V_{i}-\sum_{i=1}^{N} \frac{1}{R_{i}} \int_{0}^{V_{i}} u_{i} d V(7)$

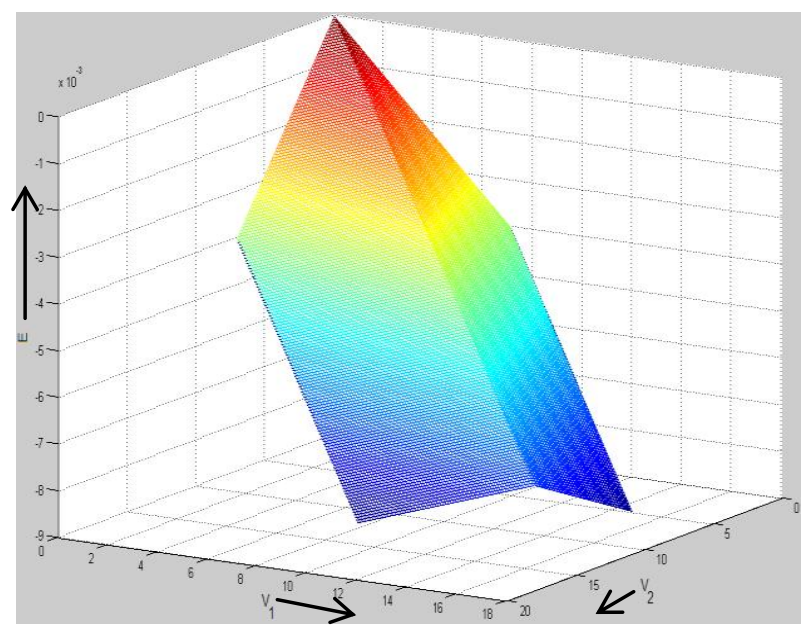

Fig 2: Plot of the second and third terms in equation (7)
The last term in (7) is usually neglected for high values of the open-loop gain of the opamp used to realize the neurons. The first term on the right hand side of (7) is quadratic which tries to minimize the number of colors. The second term has got a negative sign. Therefore, the energy function (E) will be minimized if second term is maximized. This happens when the voltages corresponding to connected nodes in a graph are far away from each other. The first two terms on the right hand side are balancing each other to color a graph properly. The third term also contributes to lowering of number of different colors by eliminating all those local minima in the energy function for which node voltages are negative. The combined effect of the second and third terms in (7) for a typical 2-node problem is shown in Fig. 2.

\section{SIMULATION RESULTS}

The proposed network was tested for various random graphs as well as a standard benchmark problem for graph coloring, myciel3.col [6]. Breadboard implementation as well as PSPICE simulation was performed. The results of the tests are given in table 1 from which it is seen that the proposed network gives a solution to all the problems tested and in all the cases the solution is very near to the chromatic number of the graph. Simulation runs of the proposed network for the myciel3.col benchmarking problem returned the best solution of 5 colors which is very near to the chromatic number for myciel3.col, that being 4. The performance of the proposed network was also compared with a network proposed earlier [4]. Table 2 gives this performance comparison. It is evident that substantial improvements have been achieved both in the best and the average solutions for most examples.

Table1.Hardware and PSPICE simulation test results for the proposed network

\begin{tabular}{|c|c|c|c|c|c|c|c|c|c|c|c|c|c|c|c|c|}
\hline \multirow{3}{*}{ s. No. } & \multirow{3}{*}{ Test Graph } & \multicolumn{12}{|c|}{ Hardware Results } & \multicolumn{2}{|c|}{ Simulation Results } & \multirow{3}{*}{$\begin{array}{c}\text { Chromatic } \\
\text { Number }\end{array}$} \\
\hline & & \multicolumn{10}{|c|}{ Voltages of different nodes with node no. } & \multirow{2}{*}{$\begin{array}{l}\text { No. of } \\
\text { colors }\end{array}$} & \multirow{2}{*}{$\begin{array}{c}\text { Frequency } \\
\text { of } \\
\text { occurrence }\end{array}$} & \multirow{2}{*}{$\begin{array}{l}\text { No. of } \\
\text { colors }\end{array}$} & \multirow{2}{*}{$\begin{array}{c}\text { Frequency } \\
\text { of } \\
\text { occurrence }\end{array}$} & \\
\hline & & 1 & 2 & 3 & 4 & 5 & 6 & 7 & 8 & 9 & 10 & & & & & \\
\hline \multirow{2}{*}{1} & & & & & & & & & & & & & & 2 & $8 / 10$ & \multirow{2}{*}{2} \\
\hline & & 0 & 3 & 0 & 3 & 0 & 3 & 0 & 3 & - & - & 2 & $25 / 25$ & 3 & $2 / 10$ & \\
\hline \multirow{2}{*}{2} & & 0 & $\boldsymbol{V}$ & 0 & $\boldsymbol{V}$ & 0 & $\boldsymbol{V}$ & 0 & $\boldsymbol{V}$ & \multirow{2}{*}{ - } & \multirow{2}{*}{ - } & 2 & $22 / 25$ & 2 & $5 / 10$ & \multirow{2}{*}{2} \\
\hline & & $\frac{V}{3}$ & 0 & $\frac{V}{3}$ & $V$ & 0 & $\boldsymbol{V}$ & $\frac{v}{3}$ & $\boldsymbol{V}$ & & & 3 & $3 / 25$ & 3 & $5 / 10$ & \\
\hline 3 & & 0 & $\boldsymbol{V}$ & 0 & $\frac{2 v}{3}$ & 0 & $\boldsymbol{V}$ & 0 & $\boldsymbol{V}$ & 0 & $\frac{2 v}{3}$ & 3 & $25 / 25$ & 3 & $10 / 10$ & 2 \\
\hline 4 & & 0 & $\boldsymbol{V}$ & 0 & $\boldsymbol{V}$ & 0 & $\boldsymbol{V}$ & 0 & $\boldsymbol{V}$ & 0 & $V$ & 2 & $25 / 25$ & 3 & $10 / 10$ & 2 \\
\hline \multirow{2}{*}{5} & \multirow{2}{*}{ Mycie } & \multirow{2}{*}{\multicolumn{10}{|c|}{ Hardware Test Not Performed }} & \multirow{2}{*}{ - } & \multirow{2}{*}{ - } & 5 & $1 / 10$ & \multirow{2}{*}{4} \\
\hline & & & & & & & & & & & & & & 6 & $9 / 10$ & \\
\hline
\end{tabular}


Table2.Performance comparison of the proposed and existing networks

\begin{tabular}{|c|c|c|c|c|c|c|}
\hline \multirow{3}{*}{$\begin{array}{l}\text { s. } \\
\text { No. }\end{array}$} & \multirow{3}{*}{ Test Graph } & \multicolumn{4}{|c|}{ Simulation Results } & \multirow{3}{*}{$\begin{array}{l}\text { Chromatic } \\
\text { Number }\end{array}$} \\
\hline & & \multicolumn{2}{|c|}{ Network of [5] } & \multicolumn{2}{|c|}{ Proposed Network } & \\
\hline & & $\begin{array}{c}\begin{array}{c}\text { Minimum } \\
\text { number of } \\
\text { colors }\end{array} \\
\end{array}$ & $\begin{array}{l}\text { Average number of } \\
\text { colors in } 10 \text { trials }\end{array}$ & $\begin{array}{c}\text { Minimum } \\
\text { number of } \\
\text { colors }\end{array}$ & $\begin{array}{l}\text { Average number of } \\
\text { colors in } 10 \text { trials }\end{array}$ & \\
\hline 1 & & 4 & 4.5 & 3 & 3 & 2 \\
\hline 2 & & 4 & 4.4 & 3 & 3 & 3 \\
\hline 3 & & 7 & 7.8 & 5 & 5.7 & 4 \\
\hline 4 & & 7 & 8.2 & 5 & 5.4 & 4 \\
\hline 5 & Myciel3.cc & 5 & 6.5 & 5 & 5.9 & 4 \\
\hline
\end{tabular}

\section{DISCUSSION}

This section deals with the monolithic implementation issues of the proposed circuit. The PSPICE simulations assumed that all operational amplifiers are identical, and therefore, analysis is required to determine how deviations from this assumption affect the performance of the network. While the component values used for the capacitors and resistors affect the convergence time, in a real implementation, they would also need to be chosen keeping in mind limitations of the active devices to source or sink currents. Effects of variations in component values from one neuron to another also need to be investigated. In fact, the drawbacks of the use of opamps in the proposed circuit suggest that a real, large scale implementation of the circuit for colouring a graph with large number of nodes might be quite different. Alternative realizations based on the differential equations (5) governing the system of neurons are being investigated. An implementation based on operational transconductance amplifiers (OTAs) would appear to be one viable alternative; similarly, circuits using MOS transistors operating in the subhreshold regime would be another. In the latter case, the $\tanh ($.$) nonlinearity is easily obtained because of the$ transistor's current-voltage characteristic [7] and is expected to be the focus of future work. However, the present manuscript focuses mainly on the principle of such a network, and a VLSI implementation is beyond the scope of this paper.

\section{CONCLUSION}

A feedback neural network based architecture to solve graph coloring problem is presented. Test results of hardware implementation and PSPICE simulation for five different graphs including the myciel3.col benchmark problem are presented. Further, PSPICE simulations of the proposed circuit show improvements in both the minimum and the average number of colors over its predecessor.

\section{REFERENCES}

[1] Zeitlhofer, T. and Wess, B. 2004. A Comparison of Graph Coloring Heuristics for Register Allocation based on Coalescing in Interval Graphs. Proc. ISCAS 04, vol. IV, $529-532$.

[2] El-Fishawy, N.A., Hadhood, M. M., Elnoubi, S., and ElSersy, W.2000. A modified Hopfield neural network algorithm for cellular radio channel assignment. Proc. TENCON 2000, 2, $213-216$.

[3] Blas, A.D., Jagota, A., and Hughey, R. 2002. Energy Function - Based Approaches to Graph Coloring. IEEE Trans. On Neural Networks, 13, 81-91.

[4] Rahman, S.A., Jayadeva and Dutta Roy, S.C. 1999. Neural network approach to graph colouring. Electronics Letters, 35(14), 1173-1175.

[5] Wu, C.W. 1998. Graph Coloring via Synchronization of Coupled Oscillators. IEEE Trans. Circuits Syst. I, 45(9), 974-978.

[6] Lui, J., Zhong, W., and Jiao, L.2006. Comments on "The 1993 DIMACS Graph Coloring Challenge" and "Energy Function-Based Approaches to Graph Coloring" IEEE Trans. On Neural Networks, 17(2), 533.

[7] Newcomb, R.W. and Lohn, J.D.1998. Analog VLSI for neural networks in The Handbook of Brain Theory and Neural Networks. Cambridge, MA: The MIT Press. 